\title{
Silicon-based waveguide-coupled planar photonic-crystal-embedded microcavities
}

\author{
Kevin K. Tsia and Andrew W. Poon \\ Department of Electrical and Electronic Engineering, The Hong Kong University of Science and Technology, \\ Clear Water Bay, Hong Kong SAR, China \\ Tel: (852)-2358-7905, fax: (852)-2358-1485, email: eeawpoon@ust.hk
}

\begin{abstract}
We numerically simulate silicon-based waveguide-coupled planar square microcavities with embedded square lattice of air holes. Our two-dimensional finite-difference time-domain simulations reveal quasi-periodic high-Q resonances with frequencies limited within the first band of the photonic crystal.
\end{abstract}

OCIS code: (230.3990) Microstructure devices, (260.5740) Resonance

Dispersion-based phenomena in photonic crystals (PCs) as an optical guiding mechanism, such as self-collimation [1] and super-prism effect [2], have been attracting considerable interest. Recently, dispersion-based PC components utilizing high refractive-index contrast square lattice of submicrometer air holes have also been proposed and experimentally demonstrated on silicon-on-insulator (SOI) substrates [3]. In this summary, we numerically simulate a new class of planar photonic structures that are comprised of a square lattice of submicrometer air holes embedded in a silicon-based square micropillar cavity. We refer to this class of photonic structures as photonic-crystal-embedded microcavities (PCEMs) [4]. Our previous work on PCEMs [4] has revealed multiple high-Q resonances with different mode field patterns. We identified modes with about two orders of magnitude intensity enhancement in the silicon core, and modes with about an order of magnitude intensity enhancement in the air holes. Here we use commercially available two-dimensional (2-D) finite-difference time-domain (FDTD) simulation tool [5] to calculate the transmission spectra and the mode-field patterns of the waveguide-coupled PCEMs. We find that the coupled modes are limited within the first band of the PC. The intensity enhancement in the air holes can approach two orders of magnitude with a larger lattice dimension.

Figure 1 (a) shows a schematic of the PCEM evanescently side-coupled with a submicrometer silicon waveguide. An $N \times N(N=6)$ 2-D square lattice of air holes with lattice periodicity $a$ and hole radius $r=0.3 a$ is embedded in a silicon square microcavity of size $L$. It has been recognized that light can be partially confined in a square microcavity by total internal reflection at the cavity sidewalls [6]. High-Q resonances can be excited only when the cavity wave satisfies the wavefront-matching condition upon traveling each cavity round trip. However, square microcavities are typically multimode [6] with round-trip ray orbits dispersed over a range of propagation directions in the bulk medium (Fig. 1 (b)). Whereas we expect PCEM resonances can be excited in a similar manner, the major advantage of PCEM is that the cavity lightwave can be coherently scattered and guided in a particular direction governed by the PC dispersion relation. Hence, almost single set of resonance round-trip ray

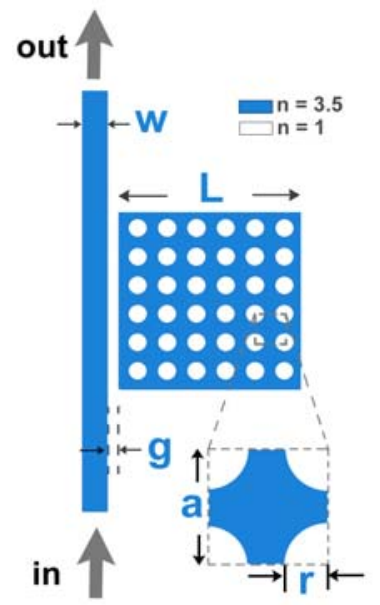

(a)

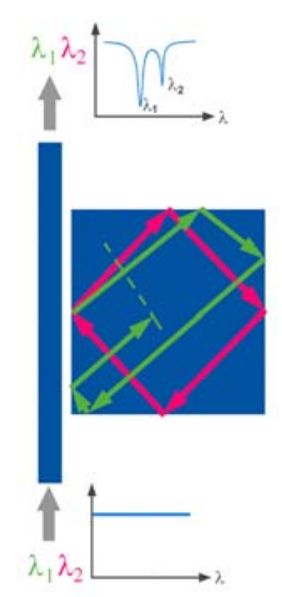

(b)

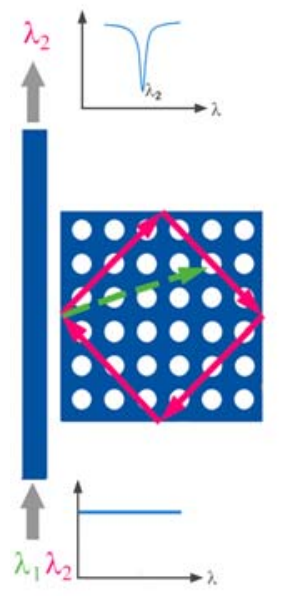

(c)

Fig.1 (a) Schematic of the PCEM comprised of a $6 \times 6$ square lattice of air holes ( $a=0.465 \mu \mathrm{m} ; r=0.3 a$ ) bounded by a silicon $(n=3.5)$ square microcavity. $(L=2.935 \mu \mathrm{m} ; w=0.375 \mu \mathrm{m} ; g=0.2 \mu \mathrm{m})$. (b), (c) Working principles of the waveguide-coupled square microcavity and the waveguide-coupled PCEM. 
orbits near the $Г \mathrm{M}$ direction is expected for PCEMs, as illustrated in Fig. 1 (c).

Figures 2 (a) and 2 (b) show the FDTD-calculated TE-polarized (E-field in-plane) transmission spectra of a waveguide-coupled 6x6 PCEM and of a waveguide-coupled square microcavity of the same $L$ as the PCEM. The PCEM transmission spectrum is nearly single-mode, while the square microcavity spectrum is multimode. For the 6x6 PCEM, only 4 pronounced quasi-periodic resonances are efficiently input coupled within the entire spectrum. The highest frequency mode $A_{6}$ has the highest $\mathrm{Q} \approx 1,200$ and a coupling efficiency of about $70 \%$. Figure 2 (c) shows the $\mathrm{A}_{6}$ mode-field pattern, which has a $6 \times 6$ lattice of field extrema confined in the air holes and evolves in a standing-wave-like manner. This type of square-lattice mode-field pattern resembles that of the square microcavity normal mode $\left(m_{x}, m_{y}\right)=(6,6)$ (Fig. $\left.2(\mathrm{e})\right)$, where $m_{x}$ and $m_{y}$ are integer numbers of field extrema along the $\mathrm{x}$ and $\mathrm{y}$ directions [6]. Mode $\mathrm{A}_{6}$ has a maximum internal intensity of about 8 times of the input intensity at the PCEM center air holes (Fig. 2 (d)), whereas the square microcavity mode $(6,6)$ only has an order of unity internal intensity enhancement (Fig. 2 (f)). We remark that Xu et al. [7] has also recently reported a similar phenomenon of localized intensity enhancement in nanometer-size low-index materials.

We also investigate the resonance transmission characteristics with a larger lattice dimension $N=11$, as shown in Fig. 3 (a). Note that the corresponding cavity size $L$ also increases. The number of coupled modes increases by a factor of three as compared with that of $\mathrm{N}=6$. The highest frequency mode $\mathrm{A}_{11}$ has a high Q-factor exceeding 7,000 with a coupling efficiency of about $95 \%$. Note that mode $A_{11}$ is very near to and below the band-edge frequency $(a / \lambda=0.219$, dashed line $)$ of the infinite PC's first band. It is expected that when $N$ increases, the dispersion characteristics approach that of an infinite PC. Figure 3 (b) shows the transmission spectrum of the square microcavity with the same $L=5.26 \mu \mathrm{m}$. The number of coupled square modes is approximately 4 times that of coupled PCEM modes within the frequency range from $a / \lambda=0.16$ to $a / \lambda=0.28$ and have resonance frequencies beyond $a / \lambda=0.219$. Figures 3 (c) and 3 (d) show that mode $A_{11}$ has a $11 \times 11$ lattice of field extrema confined in the air holes with a maximum internal intensity exceeding 90 times of the input intensity.
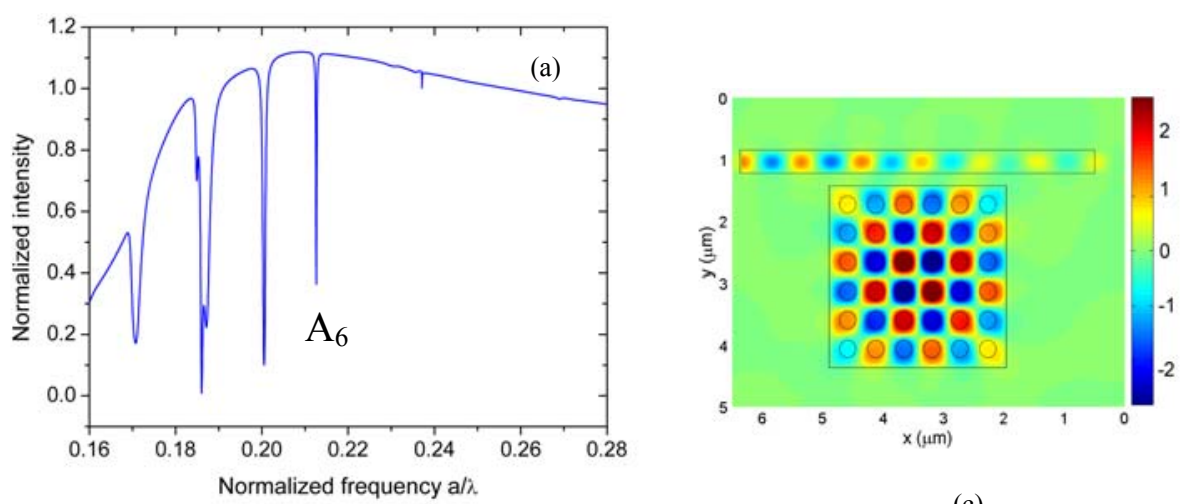

(c)

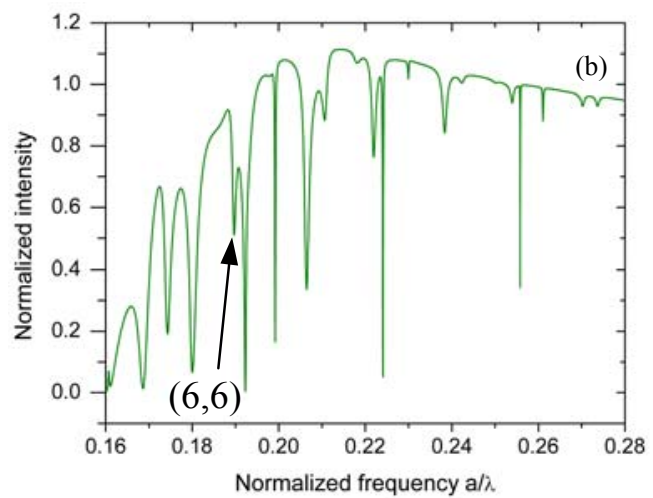

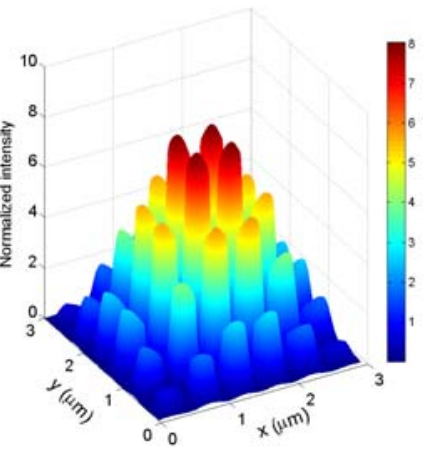

(d)

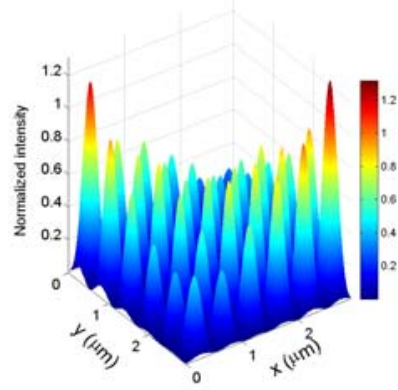

(f)

Fig.2 (a), (b) 2-D FDTD-calculated TE-polarized transmitted spectra of the 6x6 PCEM and the square microcavity with the same $L(=2.935 \mu \mathrm{m})$ as the 6x6 PCEM. (c), (d) Mode-field pattern and three-dimensional intensity profile of mode $\mathrm{A}_{6}$ of the PCEM. (e), (f) Mode-field pattern and three-dimensional intensity profile of mode $(6,6)$ of the square microcavity. 

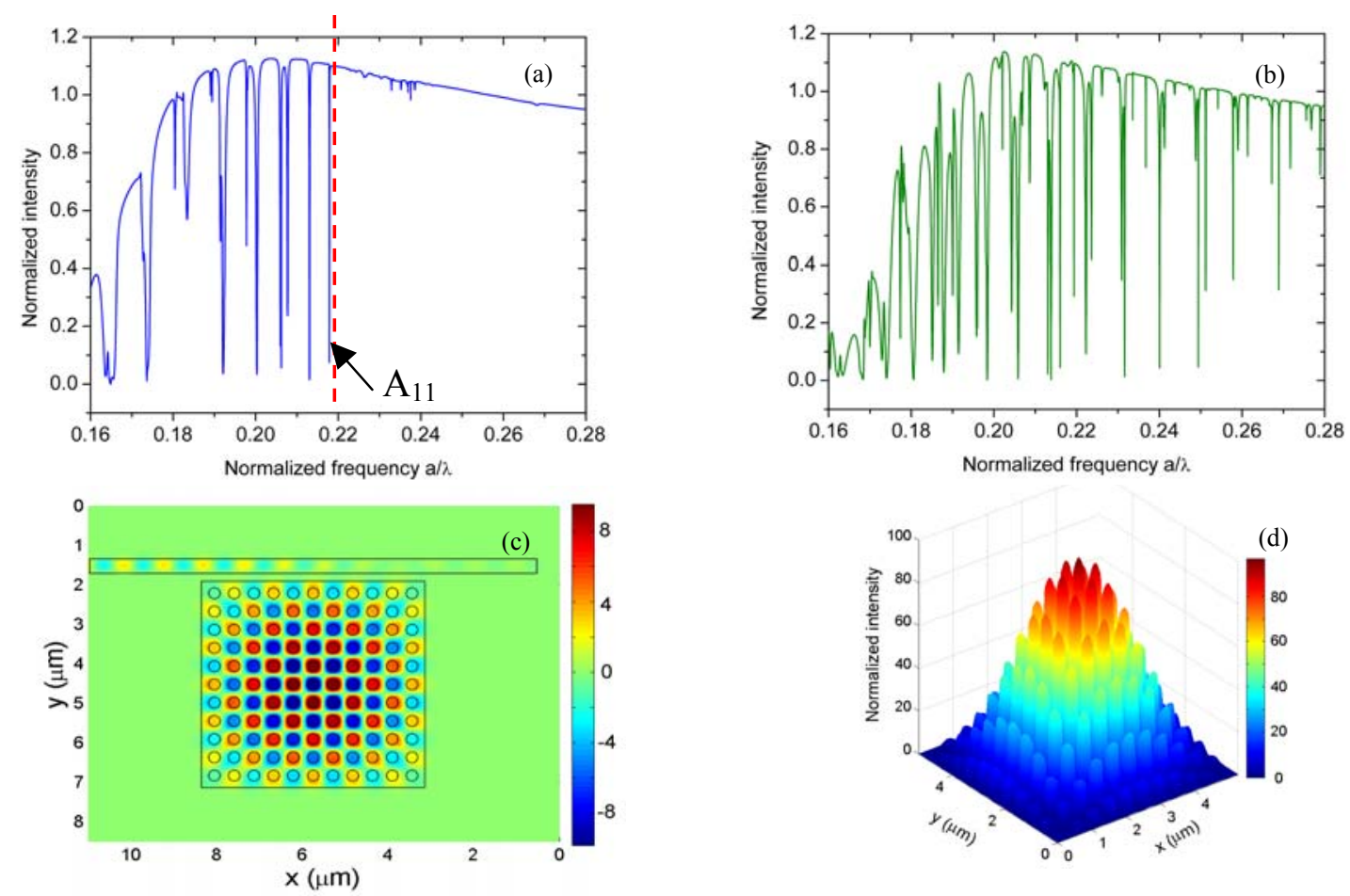

Fig. 3 (a), (b) 2-D FDTD-calculated TE-polarized transmitted spectra of the 11x11 PCEM and the square microcavity with the same $L(=5.26 \mu \mathrm{m})$ as the 11x11 PCEM. (c), (d) Mode-field pattern and three-dimensional intensity profile of mode $\mathrm{A}_{11}$ of the PCEM.

In summary, we numerically demonstrate that high refractive-index contrast waveguide-coupled PCEMs possess quasi-periodic high-Q resonance modes that are guided by the PC dispersion and partially confined by the microcavity sidewalls. The coupled modes are limited within the first PC band. The highest frequency mode near the PC bandedge displays a square lattice of mode field pattern with a large intensity enhancement in the submicrometer air holes. For a large-size PCEM, the highest frequency mode has a maximum of about two orders of magnitude intensity enhancement, a relatively large input coupling efficiency exceeding $90 \%$, and a high Q of about 7,000. At present, we are optimizing the waveguide-coupled PCEM design and fabricating the devices on thin-film silicon-on-insulator (SOI) substrates for experimental confirmation. We envision PCEMs can find important applications as WDM filters, micro-lasers, biosensors and non-linear optics devices.

The research was substantially supported by grants from the Research Grants Council and the University Grants Council of the Hong Kong Special Administrative Region, China (Project No. HKUST6166/02E, HIA01/02.EG05 \& DAG03/04.EG38).

\section{References}

[1] J. Witzens, M. Lončar, and A. Scherer, "Self-collimation in planar photonic crystals," IEEE J. Sel. Top. Quant. Electron. 8, 1246-1257 (2002)

[2] L. Wu, M. Mazilu, and T. F. Krauss, "Beam steering in planar-photonic crystals: from superprism to supercollimator," J. Lightwave Technol. 21, 561-566 (2003)

[3] D. W. Prather, S. Shi, D. M. Pustai, C. Chen, S. Venkataraman, A. Sharkawy, G. J. Schneider, J. Murakowski, "Dispersion-based optical routing in photonic crystals," Opt. Lett. 29, 50-52 (2004)

[4] K. K. Tsia and A. W. Poon, "Photonic-crystal-embedded microcavities, " presented at 2004 Conference on Lasers and Electro Optics/International Quantum Electronics Conference, San Francisco, May, 2004

[5] FullWAVE, Rsoft Inc. Research Software, http://www.rsoftinc.com

[6] C. Y. Fong and A. W. Poon, "Mode field patterns and preferential mode coupling in planar waveguide-coupled square microcavities," Opt. Express 11, 2897-2904 (2003)

[7] Q. Xu, V. R. Almeida, C. A. Barrios, R. R. Panepucci, and M. Lipson, "Silicon void nano-waveguides for guiding and confining light," presented at 2004 Conference on Lasers and Electro Optics/International Quantum Electronics Conference, San Francisco, May, 2004 